\section{Part 4. Post-cardiac arrest care: 2015 Korean Guidelines for Cardiopulmonary Resuscitation}

\author{
Young-Min Kim ${ }^{1}$, Kyu Nam Park', Seung Pill Choi ${ }^{1}$, Byung Kook Lee², \\ Kyungil Park ${ }^{3}$, Jeongmin Kim ${ }^{4}$, Ji Hoon Kim', Sung Phil Chung ${ }^{5}$, \\ Sung Oh Hwang ${ }^{6}$
}

\begin{abstract}
1Department of Emergency Medicine, The Catholic University of Korea College of Medicine, Seoul, Korea ${ }^{2}$ Department of Emergency Medicine, Chonnam National University Medical School, Gwangju, Korea ${ }^{3}$ Department of Internal Medicine, Dong-A University College of Medicine, Busan, Korea ${ }^{4}$ Department of Anesthesiology and Pain Medicine, Yonsei University College of Medicine, Seoul, Korea ${ }^{5}$ Department of Emergency Medicine, Yonsei University College of Medicine, Seoul, Korea

${ }^{6}$ Department of Emergency Medicine, Yonsei University Wonju College of Medicine, Wonju, Korea
\end{abstract}

High quality integrated post-cardiac arrest care can significantly influence the outcome of patients with cardiac arrest, especially neurological recovery, and numerous studies have been conducted on this topic. In the 2015 Korean cardiopulmonary resuscitation (CPR) guidelines, post-cardiac arrest care was emphasized with effective advanced life support as one of the essential chains for survival. Based on an in-depth scientific evidence review using a standardized methodological approach proposed by the GRADE (Grading of Recommendations Assessment, Development, and Evaluation) working group, the guidelines either partially updated the recommendations or added new recommendations for each specific topic.

\section{POST-CARDIAC ARREST CARE STRATEGIES (Fig. 1 and Table 1)}

\section{Airway and breathing}

When a patient is unconscious after the return of spontaneous circulation (ROSC), the airway should be secured by tracheal intubation, and this should be confirmed by measuring the endtidal carbon dioxide $\left(\mathrm{ETCO}_{2}\right)$ and oxygen saturation $\left(\mathrm{SpO}_{2}\right)$ levels using waveform capnography and pulse oximetry; additionally, mechanical ventilation should be performed while continuously monitoring the $\mathrm{ETCO}_{2}$ and $\mathrm{SpO}_{2}$. To avoid hypoxia, it is reasonable to use the highest available oxygen concentration until appropriate monitoring is available to evaluate the arterial oxygen tension $\left(\mathrm{PaCO}_{2}\right)$ or arterial oxygen saturation $\left(\mathrm{SaO}_{2}\right)$ levels. When resources are available to titrate the fraction of inspired oxygen $\left(\mathrm{FiO}_{2}\right)$ and monitor the saturation, it is reasonable to decrease the $\mathrm{FiO}_{2}$, provided that the $\mathrm{SaO}_{2}$ level can be maintained at the target range.

A study based on a registry reported that hyperoxia within 24 hours after ROSC was associated with a poor outcome compared to hypoxemia or normoxemia within 24 hours. ${ }^{1}$ In another study, hyperoxia was shown to have a dose-dependent relationship with poor outcomes, rather than with a certain threshold level. ${ }^{2}$ In addition, hyperoxia was associated with poor prognosis in a study of patients with cardiac arrest who had received mild therapeutic hypothermia. ${ }^{3}$ In contrast, a study of approximately 12,000 patients with cardiac arrest reported that there was no association between hyperoxia and mortality after the inspired oxygen level and disease severity
elSSN: 2383-4625

Received: 16 February 2016

Revised: 19 March 2016

Accepted: 19 March 2016

Correspondence to: Young-Min Kim Department of Emergency Medicine, Seoul St. Mary's Hospital, The Catholic University of Korea College of Medicine, 222 Banpo-daero, Seocho-gu, Seoul 06591, Korea

E-mail:emart@catholic.ac.kr

Kyu Nam Park

Department of Emergency Medicine, Seoul St. Mary's Hospital, The Catholic University of Korea College of Medicine, 222 Banpo-daero, Seocho-gu, Seoul 06591, Korea

E-mail:emsky@catholic.ac.kr

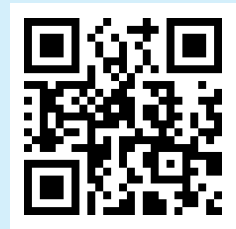

How to cite this article: Kim YM, Park KN, Choi SP, Lee BK, Park K, Kim J, Kim JH, Chung SP, Hwang SO. Part 4. Post-cardiac arrest care: 2015 Korean Guidelines for Cardiopulmonary Resuscitation. Clin Exp Emerg Med 2016;3(S):S27-S38.

This is an Open Access article distributed under the terms of the Creative Commons Attribution Non-Commercial License (http:// creativecommons.org/licenses/by-nc/3.0/). 


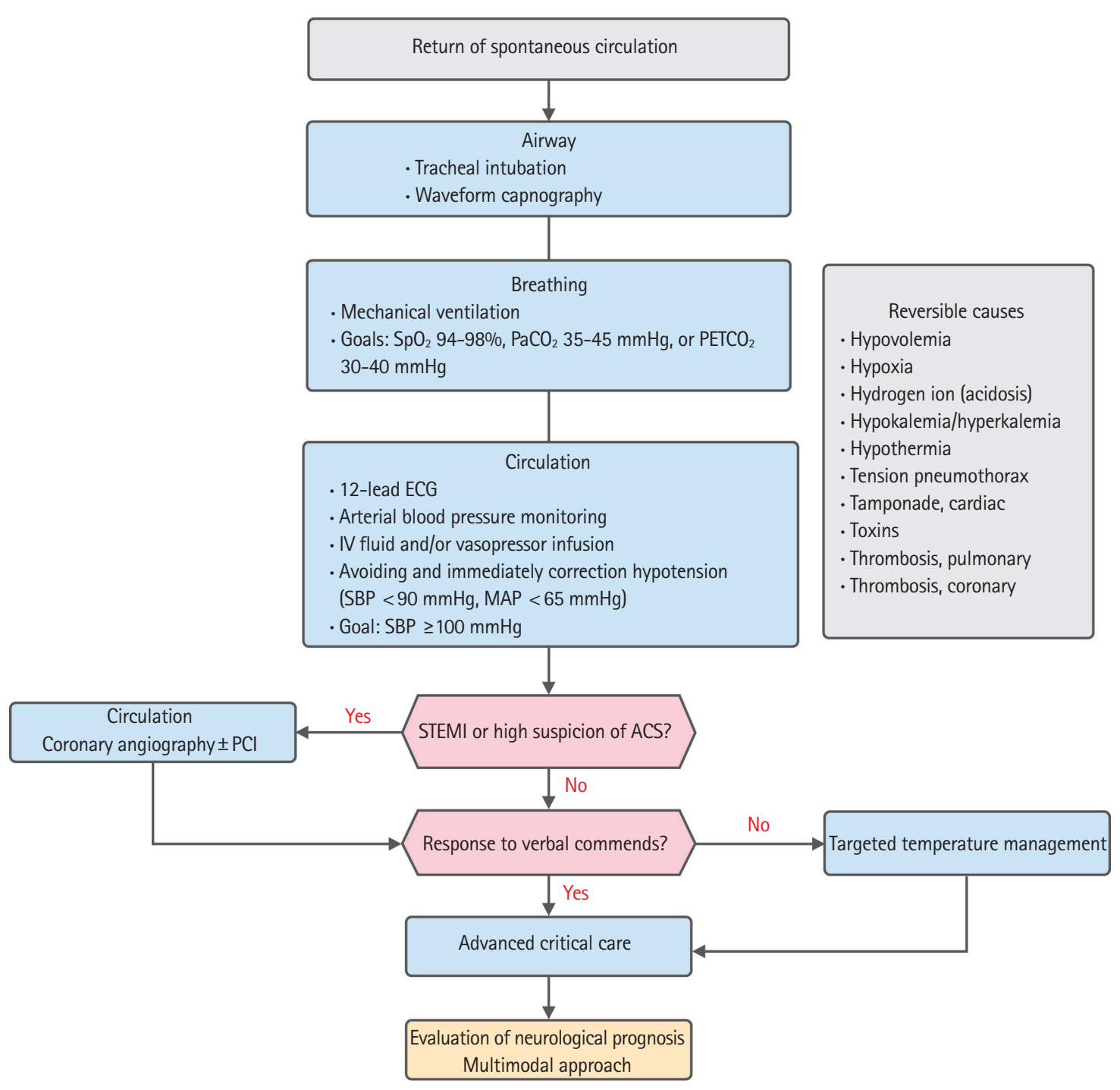

Fig. 1. Post-cardiac arrest care algorithm. $\mathrm{SpO}_{2}$, oxygen saturation; $\mathrm{PaCO}_{2}$, arterial oxygen tension; $\mathrm{PETCO}_{2}$, partial pressure of end-tidal carbon dioxide; ECG, electrocardiography; IV, intravenous; SBP, systolic blood pressure; MAP, mean arterial pressure; PCl, percutaneous coronary intervention; STEMI, STelevation myocardial infarction; ACS, acute coronary syndrome.

were adjusted. ${ }^{4}$ A meta-analysis of 14 observational studies showed significant heterogeneity across studies. ${ }^{5}$ Therefore, it is suggested to maintain the $\mathrm{SaO}_{2}$ at a level of $94 \%$ to $98 \%$ to avoid hypoxemia and the potential risk of hyperoxia.

Hypocapnia causes cerebral vasoconstriction, and it reduces cerebral blood flow. ${ }^{6}$ Observational studies using a registry of patients with cardiac arrest have reported an association between hypocapnia and poor neurologic outcome. ${ }^{7.8}$ Two observational studies reported that mild hypercapnia was associated with more favorable neurologic outcomes in patients with cardiac arrest in intensive care units. ${ }^{79}$ However, several other studies did not show a consistent association between hypercapnia and outcome. ${ }^{7-10}$ Therefore, it is suggested to maintain the $\mathrm{CO}_{2}$ level within a normal physiological range $\left(\mathrm{PaCO}_{2} 35\right.$ to $45 \mathrm{mmHg}$ or $\mathrm{ETCO}_{2} 30$ to $40 \mathrm{mmHg}$ ).

\section{Circulation}

\section{1) Hemodynamic stabilization}

Post-cardiac arrest patients are often hemodynamically unstable due to the underlying etiology of arrest, myocardial dysfunction, and systemic ischemia/reperfusion response. ${ }^{11}$ Therefore, immediately after ROSC, an arterial catheter should be promptly inserted, and the arterial blood pressure should be monitored continuously. If an arterial catheter cannot be inserted, the blood pressure should be frequently measured noninvasively until the patient becomes hemodynamically stable. Dobutamine can be helpful for postcardiac arrest myocardial dysfunction. ${ }^{12}$ In addition, vasodilation occurs due to the systemic ischemia/reperfusion response; thus, dopamine or norepinephrine may be needed, and intravenous fluids can be an effective treatment depending on the situation. ${ }^{11}$ If 
Table 1. The strategies in adult immediate post-cardiac arrest care

\begin{tabular}{|c|c|}
\hline Strategy & Doses/details \\
\hline Airway & $\begin{array}{l}\text { Consider tracheal intubation and waveform } \\
\text { capnography }\end{array}$ \\
\hline Breathing-oxygenation & $\begin{array}{l}\text { Avoid hypoxemia } \\
\text { Goal: titrate } \mathrm{FiO}_{2} \text { to achieve } \mathrm{SpO}_{2} 94 \% \text { to } 98 \%\end{array}$ \\
\hline Breathing-ventilation & $\begin{array}{l}\text { Avoid excessive ventilation } \\
\text { Goal: titrate to target } \mathrm{PaCO}_{2} 35 \text { to } 45 \mathrm{mmHg} \text { or } \\
\text { PETCO }_{2} 30 \text { to } 40 \mathrm{mmHg}\end{array}$ \\
\hline Circulation-hemodynamics & $\begin{array}{l}\text { Avoiding and immediately correcting hypotension } \\
\text { (systolic blood pressure }<90 \mathrm{mmHg} \text {, mean arte- } \\
\text { rial pressure }<65 \mathrm{mmHg} \text { ) } \\
\text { Goal: systolic blood pressure } \geq 100 \mathrm{mmHg}\end{array}$ \\
\hline Circulation-vasoactive drugs & $\begin{array}{l}\text { Norepinephrine: } 0.1 \text { to } 0.5 \mathrm{mcg} / \mathrm{kg} / \mathrm{min} \\
\text { Dopamine: } 5 \text { to } 10 \mathrm{mcg} / \mathrm{kg} / \mathrm{min} \\
\text { Epinephrine: } 0.1 \text { to } 0.5 \mathrm{mcg} / \mathrm{kg} / \mathrm{min}\end{array}$ \\
\hline Correct the reversible causes & $\begin{array}{l}\text { Hypovolemia, hypoxia, hydrogen ion (acidosis), } \\
\text { hypokalemia/hyperkalemia, hypothermia, ten- } \\
\text { sion pneumothorax, cardiac tamponade, toxins, } \\
\text { pulmonary thrombosis, coronary thrombosis }\end{array}$ \\
\hline
\end{tabular}

$\mathrm{FiO}_{2}$, fraction of inspired oxygen; $\mathrm{SpO}_{2}$, oxygen saturation; $\mathrm{PaCO}_{2}$, arterial oxygen tension; $\mathrm{PETCO}_{2}$, Partial pressure of end-tidal carbon dioxide.

hemodynamic instability persists even with the infusion of intravenous fluids or vasoactive drugs, a mechanical circulatory assistance device should be considered. ${ }^{13}$

Although there are observational studies of patients with cardiac arrest that have investigated the relationship between blood pressure and outcome, a controlled study has not been conducted on a target goal of blood pressure. An observational study examined whether treatment with a specific hemodynamic goal (e.g., a mean arterial pressure [MAP] $>65 \mathrm{mmHg}$ ) would improve neurologic and functional outcome compared to treatment without a specific hemodynamic goal. The study reported that the mortality rate was higher and the functional recovery was lower in the patient group whose systolic blood pressure (SBP) was $<90 \mathrm{mmHg}$ after CPR compared to other patient groups whose SBP was $\geq 90$ $\mathrm{mmHg} \cdot{ }^{14}$ Two retrospective studies reported that the survival rate decreased in patients who maintained an SBP $<90 \mathrm{mmHg}$ and $<100 \mathrm{mmHg} \cdot{ }^{15,16}$ As several before-and-after studies have implemented a bundle of care, which included a blood pressure goal, the effect of blood pressure cannot be evaluated alone. Moreover, different studies have shown different results regarding a specific level of blood pressure, and the level of evidence from existing studies is insufficient to determine a target blood pressure goal.

Seven studies have investigated the effect of a bundle of care on neurologic outcome, and they reported different results. Some studies have found no association between a specific target blood pressure and neurologic outcome. In one of the studies, a MAP $>80 \mathrm{mmHg}$ was a goal, and in another study, an intervention was performed when a goal of MAP was below $75 \mathrm{mmHg}$, and both of which reported that using a bundle of care, including a hemodynamic goal, was not associated with the neurologic outcome. ${ }^{17,18}$ In contrast, other studies have found that a bundle of care that included a blood pressure goal improved the neurologic outcome. Two prospective observational studies have reported that maintaining a MAP $>65 \mathrm{mmHg}$ improved the neurologic outcome, and an additional study demonstrated that the neurologic outcome was better in the patient group who maintained a MAP $>100 \mathrm{mmHg}$ at 2 hours after ROSC, suggesting an association between MAP and the neurological outcome. ${ }^{19-21}$ Yet, another study reported that in patients with a good neurologic outcome, maintaining the time-weighted MAP over $70 \mathrm{mmHg}$ was associated with the neurologic outcome. ${ }^{22}$ Two before-and-after observational studies have investigated the effect of using a bundle of care on the survival rate. Both of these studies used a bundle with a MAP $>80 \mathrm{mmHg}$ and $>65 \mathrm{mmHg}$ as a goal, but there was no significant difference in the survival rate..$^{17,20}$

Thus, the evidence is insufficient to determine a specific hemodynamic goal for post-cardiac arrest care. It is suggested that hypotension (SBP $<90 \mathrm{mmHg}$ or MAP $<65 \mathrm{mmHg}$ ) should be immediately corrected, and a hemodynamic goal should be determined for individual patients, while maintaining an SBP $>100$ $\mathrm{mmHg}$.

\section{2) Evaluation and treatment of reversible causes}

After ROSC and during CPR, the resuscitation team should make efforts to evaluate the reversible causes of cardiac arrest (i.e., the five H's and five T's: hypovolemia, hypoxia, hydrogen ions [acidosis], hyper/hypokalemia, hypothermia, thromboembolism, thrombosis, tension pneumothorax, cardiac tamponade, and tablets) and then treat the patient.

\section{(1) Intervention of acute coronary syndromes}

Acute coronary syndromes are a common cause of adult out-ofhospital cardiac arrest (OHCA) with no obvious extracardiac cause of arrest. ${ }^{23,24}$ Thus, a 12-lead electrocardiography (ECG) and a cardiac marker test should be obtained as soon as possible after ROSC to confirm the presence or absence of acute coronary syndromes. ${ }^{25,26}$ Coronary angiography should be performed emergently for patients with OHCA with suspected cardiac etiology and ST elevation on ECG, regardless of whether the patient is conscious. ${ }^{27-29}$ If acute coronary syndromes are highly suspected in patients with OHCA with suspected cardiac etiology but their ECG does not show ST elevation after ROSC, early coronary angiography should be considered, regardless of the consciousness status. $^{30,31}$ 
(2) Treatment of pulmonary embolism

When cardiac arrest due to pulmonary embolism is strongly suspected, chest computed tomography (CT) should be performed, if possible. If cardiac arrest due to pulmonary embolism is confirmed, thrombolytics can be administered, or surgical or percutaneous embolectomy can be performed. ${ }^{32-34}$

\section{TREATMENTS FOR OPTIMIZING NEUROLOG- ICAL RECOVERY}

\section{Temperature control}

1) Prevention and treatment of hyperpyrexia

Observational studies have reported an association between poor outcome and fever after ROSC in patients without targeted temperature management (TTM). ${ }^{35,36}$ Hyperpyrexia occurs in many patients after TM. However, the optimal approach to subsequent TTM remains unknown. Several studies have reported conflicting conclusions regarding the association with outcome. ${ }^{36-38}$ Although the effect of hyperpyrexia on the outcome of post-cardiac arrest patients is not proven, a poor outcome was associated with hyperpyrexia in comatose patients in whom cerebral damage was due to other causes such as cerebral hemorrhage or infarct, and it is relatively easy to treat or prevent hyperthermia. ${ }^{39,40}$ Therefore, it is suggested to continuously prevent or treat fever in adult comatose patients after ROSC from cardiac arrest, regardless of whether the patient received TTM.

\section{2) $\pi M$}

(1) Indications and target temperature

One randomized controlled trial (RCT) and a pseudo-randomized trial demonstrated that $T \mathrm{M}$ of $32^{\circ} \mathrm{C}$ to $34^{\circ} \mathrm{C}$ improved the neurological outcome at hospital discharge and at 6 months in adult patients with OHCA with a shockable cardiac rhythm. ${ }^{41,42}$ Therefore, OHCA with a shockable rhythm is a main indication for TTM. Regarding patients with OHCA with a non-shockable rhythm, no RCT was available. In a cohort study with a very low level of evidence, there was an association between mild induced hypothermia and 6-month survival in patients with OHCA with a nonshockable rhythm. However, a meta-analysis of two other cohort studies did not determine an association between mild induced hypothermia $\left(32^{\circ} \mathrm{C}\right.$ to $\left.34^{\circ} \mathrm{C}\right)$ and an improvement in neurological results. ${ }^{43-46}$ For patients with in-hospital cardiac arrest, no RCT has been published. A retrospective cohort study of 1,836 patients found no association between mild induced hypothermia and survival or a functionally favorable status at hospital discharge. ${ }^{47}$ However, in this study, the implementation rate of TM was very low, and the overall outcome of patients treated without TTM was poor; therefore, the aggressive implementation of TTM became necessary. Additionally, ultra-mild hypothermia $\left(36^{\circ} \mathrm{C}\right)$ has been suggested as another target temperature since a large, wellconducted RCT compared the target temperature levels of $33^{\circ} \mathrm{C}$ and $36^{\circ} \mathrm{C}$, as it found that the neurological outcome and survival at 6 months after ROSC were not improved when the temperature was controlled at $36^{\circ} \mathrm{C}$ versus $33^{\circ} \mathrm{C}^{48}$

Based on the scientific evidence, regardless of the initial rhythm for $\Pi M$, it is recommended to select and maintain a constant temperature between $32^{\circ} \mathrm{C}$ and $36^{\circ} \mathrm{C}$ in adult patients with cardiac arrest who do not show a meaningful response to verbal commands after ROSC. When making this recommendation, the writing group considered the characteristics of the cardiac arrest population in Korea and that pulseless electrical activity or asystole (i.e., a non-shockable cardiac rhythm) is relatively more frequently observed as the initial arrest rhythm. However, it is unknown whether $\Pi \mathrm{MM}$ with mild induced hypothermia $\left(32^{\circ} \mathrm{C}\right.$ to $34^{\circ} \mathrm{C}$ ) or ultra-mild hypothermia $\left(36^{\circ} \mathrm{C}\right)$ is helpful to specific subgroups with cardiac arrest, thus additional studies are needed to shed light on this issue.

\section{(2) Initiation and duration of TTM}

Neuronal injury following transient global brain ischemia/reperfusion progresses for several days in a complex biochemical cascade. Mild induced hypothermia influences various stages during the process. ${ }^{49,50}$ In particular, oxidative stress, increased excitatory amino acids, and energy depletion occur immediately after ROSC and during cardiac arrest. Theoretically, it would be helpful if mild hypothermia is induced in patients with cardiac arrest as quickly as possible. ${ }^{51,52}$ For this reason, several studies have compared the prehospital induction of hypothermia and hospital induction of hypothermia. In all seven RCTs with a medium level of evidence, there was no significant difference between the groups in terms of a poor neurologic outcome or mortality. ${ }^{53-59}$ A meta-analysis of seven studies also did not show differences in the mortality rate or poor neurologic outcome between the groups at hospital discharge. ${ }^{46}$ Accordingly, based on current scientific evidence, it cannot be concluded that the prehospital induction of TTM is better than induction at the hospital; hence, more conclusive follow-up studies are needed.

A high quality interventional study is not yet available with which to determine an optimal duration of TTM after cardiac arrest. Therefore, based on the duration used in two of the largest aforementioned RCTs, it is reasonable to maintain TTM for at least 24 hours after achieving the target temperature. ${ }^{41,48}$ 
(3) Methods of inducing and/or maintaining TTM

Diverse cooling methods are used to induce and maintain a target temperature, but there is no one best method. External surface cooling methods used widely in the past (e.g., a cooling blanket, ice packs, and a wet towel) are easy and convenient to use. However, they take longer to lower the patient's core temperature, and they are difficult to maintain at a constant level, which adds to the medical staff's high workload. Recently, body temperature control has become easier, as cooling equipment that use endovascular catheters and external cooling equipment that regulates temperature with an auto-feedback system have become available, but they are expensive. Hence, medical staff that plan to perform TTM should consider a variety of factors (e.g., the place to initiate the treatment, staff's ability and experience, speed in induction, stability during maintenance and rewarming, mobility of the equipment, adverse events associated with specific equipment or techniques, convenience of using the equipment, cost, etc.), and the most optimal cooling technique should be chosen for individual patients. ${ }^{60}$

Cold crystalloid intravenous fluid infusion is relatively easy to induce hypothermia, and it has been widely used in prehospital settings or during resuscitation for convenience. However, according to a meta-analysis of four RCTs, when TTM was initiated in the prehospital setting using cold crystalloid intravenous fluid infusion, the risk for re-arrest increased. ${ }^{46}$ In addition, pulmonary edema was increased according to one large-scale RCT. ${ }^{58}$ Therefore, it is not recommended to perform routine prehospital cooling of a patient after ROSC with the rapid infusion of cold intravenous fluid.

When TTM is performed, the patient's core temperature should be continuously monitored in the esophagus, bladder, or pulmonary artery. The pulmonary artery is the most accurate, but it has a limitation because it requires an invasive procedure. The axillary temperature or oral temperature is not appropriate for measuring changes in the core temperature, and tympanic temperature sensors are difficult to use for a long time and they are often inaccurate. The rectal temperature is commonly used, but there can be temperature gaps when hypothermia is induced rapidly; thus, careful monitoring is needed. ${ }^{61}$

Moreover, there is not enough evidence for an optimal speed of rewarming. Accordingly, it is suggested to rewarm at a speed of $0.25^{\circ} \mathrm{C}$ to $0.5^{\circ} \mathrm{C}$ per hour, which has been used often in previous studies, ${ }^{41,42,48}$ and maintain normothermia $\left(36.5^{\circ} \mathrm{C}\right.$ to $\left.37.5^{\circ} \mathrm{C}\right)$ up to 72 hours after ROSC in comatose patients even after normothermia is achieved.

\section{Glucose control}

Hyperglycemia is associated with mortality and a poor neurologic outcome in patients who have been resuscitated from cardiac arrest, and it should be appropriately controlled. There is little evidence about a target blood glucose level to improve the outcome of patients with cardiac arrest. An RCT found no difference in the 30-day mortality rate between the groups with a target blood glucose level of 72 to $108 \mathrm{mg} / \mathrm{dL}$ and 108 to $144 \mathrm{mg} / \mathrm{dL}^{62}$ In a before-and-after study that used a bundle of care with a target blood glucose level of 90 to $144 \mathrm{mg} / \mathrm{dL}$, the neurological outcome improved after implementing the bundle, but the effect could not be attributed only to the controlled blood glucose level. ${ }^{18}$ Applying the findings of these studies to cardiac arrest patients may not be appropriate, because they examined the effect of controlling blood glucose level in critically ill patients. ${ }^{63,64}$ Although is still controversy on how to control the blood glucose level in critically ill patients, the strict control of blood glucose was associated with an increased occurrence of hypoglycemia. ${ }^{62}$ Therefore, the target range of 144 to $180 \mathrm{mmHg}$ is suggested to prevent hypoglycemia, although evidence so far is insufficient. Hyperglycemia $>180 \mathrm{mmHg}$ should be treated with an insulin infusion per the hospital's protocol, and care should be taken to prevent hypoglycemia ( $<80 \mathrm{mg} / \mathrm{dL}$ ). If hypoglycemia occurs, it should be immediately corrected by administering a glucose solution. The blood glucose level, especially, fluctuates during the induction or rewarming period; therefore, it is desirable to frequently test the patient's blood glucose level.

\section{Control of seizures}

No study has directly compared patients treated for seizure with those not treated for seizure. Furthermore, there is no evidence thus far that a specific anticonvulsant or combination therapy with anticonvulsant drugs is helpful in comatose patients after cardiac arrest. Therefore, if seizure occurs or is suspected, an electroencephalogram (EEG) needs to be performed immediately to determine the presence of epileptiform activity. Non-convulsive seizures can occur while TTM is performed with a neuromuscular blocking drug; thus, if possible, an EEG should be continuously monitored, and the arterial blood gas level or change in vital signs (e.g., unexplained tachycardia) should be carefully observed. ${ }^{65}$ To control seizures, any commonly used anticonvulsant needs to be promptly injected. In a case of generalized seizure, benzodiazepine, phenytoin, sodium valproate, propofol, levetiracetam, phenobarbital, and similar drugs can be used alone or in combination with each other. In cases of myoclonus, clonazepam, sodium valproate, levetiracetam, propofol, etc. can be used alone or in combination with each other. ${ }^{66}$ Post-anoxic myoclonic status epilepti- 
cus may not respond well to anticonvulsants. ${ }^{67,68}$ Available evidence does not support the prophylactic administration of anticonvulsants. Therefore, the routine use of prophylactic anticonvulsants in post-cardiac arrest patients is not recommended.

\section{Sedation}

In most post-cardiac arrest patients, mechanical ventilation should be maintained during post-cardiac arrest care, and sedatives or analgesics may have to be intermittently or continuously administered. If a patient is sedated while TM is performed, the time required to reach to the target temperature can be reduced because shivering is prevented or reduced. Using a sedation protocol can be helpful in these cases.

Sedation after ROSC is a commonly used treatment method, but the level of evidence is not sufficient to make a recommendation about the duration to administer a sedative or neuromuscular blocker in post-cardiac arrest patients. A meta-analysis of 44 studies reported on sedative drugs that were used while TTM was performed among 68 intensive care units in various countries found that a large variety of drugs were being used. ${ }^{69}$ From the analysis, it is impossible to know which drugs may be associated with outcome, but mainly the combination therapy of an opioid and sedative was used. It is recommended to maintain a sedative over a short duration of action as much as possible, but no study has analyzed the effect of using sedatives in patients after cardiac arrest or has suggested treatment strategies. One study has suggested that the continuous administration of neuromuscular blockers may be associated with a low mortality rate. ${ }^{70}$ However, neuromuscular blockers interfere with clinical examinations, and they obscure the occurrence of seizure. Therefore, if a neuromuscular blocker is continuously administered, the EEG should be continuously monitored.

\section{EVALUATION OF NEUROLOGICAL PROGNOSTICATION}

In the 2015 guidelines, studies of patients who had and had not received TTM were evaluated. These studies evaluated the diagnostic accuracy of clinical examination, electrophysiological studies, biomarkers, and imaging tests for predicting poor neurological outcome, and they recommended diagnostic tests with a false positive rate (FPR) close to $0 \%$ and a narrow 95\% confidence interval ( $0 \%$ to $10 \%)$ as predictors.

It is recommended to predict the neurologic outcome at least 72 hours after ROSC, particularly because in most cases, a sedative and a neuromuscular blocker are administered to comatose patients after cardiac arrest while TMM is performed. An addition- al recommendation is to use a multimodal approach rather than relying on a single test or finding.

\section{Clinical examination}

For a clinical examination to predict a poor neurologic outcome within 72 hours after ROSC in comatose patients after cardiac arrest, the bilateral absence of pupillary light reflex, or the combination of the absence of pupillary light reflex and absence of corneal reflex is required in both patients with TTM (FPR 0 [0\% to 3\%]) and without TTM (FPR 0 [0\% to 8\%]). ${ }^{71-79}$

The lack of motor movement (M1) or extensor posturing (M2) to pain has a high FPR level (27\% [12\% to 48\%]) for predicting a poor neurologic outcome in comatose patients who did not receive TTM after cardiac arrest, and a similar finding was observed in patients who received TMM. ${ }^{71,73-82}$ Therefore, it is suggested to not use motor movement to pain alone to predict poor neurologic outcomes. Although they have a high sensitivity level (74\% [68\% to $79 \%]$ ), they can be used to confirm a patient's poor neurologic outcome or predict a poor neurologic outcome in combination with other predictors.

Myoclonus in comatose patients within 72 hours after cardiac arrest is associated with a high FPR (10\% to 15\%) for predicting poor neurologic outcomes; thus, it is suggested to not use it alone. In contrast, status myoclonus, which occurs within 72 hours after cardiac arrest, predicts poor neurologic outcome with a high accuracy in cases with TTM (FPR 0\% [0\% to 4\%]) and without TTM (FPR 0\% [0\% to 5\%])..$^{65,67,71,72,83,84}$ Therefore, it is useful when used in combination with other predictors. If there is residual sedation and paralysis still remains, the clinical examination can be delayed to minimize the possibility of false positives. Seventy-two hours after ROSC is suggested as the earliest time to predict a poor neurologic outcome.

\section{Electrophysiological studies}

To predict poor neurologic outcome in comatose patients after cardiac arrest, regardless of using $\Pi \mathrm{M}$, the use of the bilateral absence of $\mathrm{N} 20$ waveform recorded from somatosensory evoked potentials (SSEPS) (FPR 1\% [0\% to 3\%]) is recommended 24 to 72 hours after cardiac arrest or after rewarming. ${ }^{72-75,80-82,85-89} \mathrm{An}$ SSEP recording requires appropriate skills and experience, and efforts should be considered to avoid muscle artifacts or electrical interference from the environment of the emergency room or intensive care unit.

A lack of EEG background reactivity can accurately predict poor neurologic outcome in comatose patients after cardiac arrest during TTM (FPR 2\% [1\% to 7\%]) and within 72 hours after ROSC (FPR 0\% [0\% to 3\%]). ${ }^{80,82,90,91}$ However, using the pattern 
of background reactivity on EEG has limitations, because they are operator dependent, unquantifiable, and lack standardization. Status epilepticus (i.e., a persistent seizure over 72 hours) is commonly associated with a poor neurologic outcome (FPR 0\% to $6 \%$ ), if it occurs during hypothermia or rewarming in patients who received TMM. ${ }^{65,92}$ EEG burst-suppression can show a recovery of consciousness, if it occurs for 24 to 48 hours after ROSC in patients who did not receive $\Pi \mathrm{M}$, or during hypothermia in patients who received $\Pi \mathrm{M}_{1}{ }^{82,93}$ but persistent burst-suppression that occurs 72 hours after ROSC is always associated with a poor neurologic outcome. ${ }^{72,94}$ Therefore, it is suggested to use EEG predictors (e.g., the lack of EEG response to external stimulation, EEG burst-suppression after rewarming, and status epilepticus) after 72 hours after ROSC in combination with other predictors to predict a poor neurologic outcome in comatose patients after cardiac arrest, regardless of using TM.

To predict poor neurologic outcomes of patients who received
TTM, burst-suppression observed in the continuous amplitude-integrated EEG recordings, status epilepticus, and lack of normal trace over 36 hours can be used in combination with other predictors. ${ }^{95}$

\section{Biomarkers}

A high level or increasing levels of neuron-specific enolase (NSE) measured at 48-72 hours after ROSC can be used in combination with other predictors to predict poor neurologic outcomes of comatose patients who received TM after cardiac arrest. ${ }^{96-98} \mathrm{~A}$ careful approach is necessary when testing NSE to avoid a false positive result that can occur due to hemolysis, and it is recommended to sample various points in time as much as possible. Since different studies have reported different NSE thresholds to predict a poor neurologic outcome with $0 \%$ FPR, it is recommended to not use the serum levels of NSE and S100B alone.

Recommended timing of prognostic tests
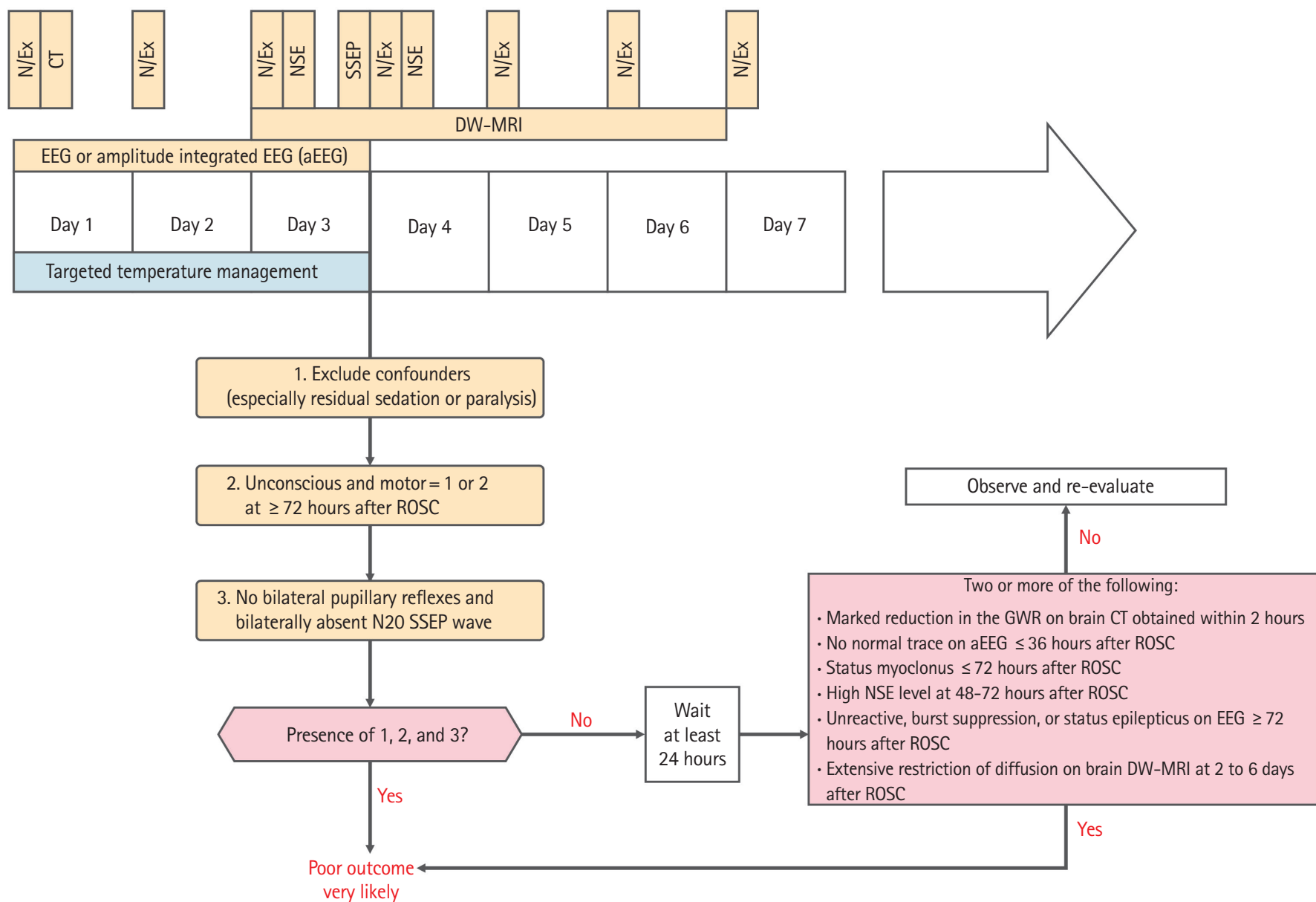

Fig. 2. Neurological prognostication algorithm. N/Ex, neurological examination; CT, computed tomography; NSE, neuron specific enolase; SSEP, somatosensory evoked potential; DW-MRI, diffusion-weighted magnetic resonance imaging; EEG, electroencephalography; ROSC, return of spontaneous circulation; GWR, gray matter/white matter ratio; CT, computed tomography; aEEG, amplitude-integrated electroencephalography. 


\section{Imaging tests}

Global cerebral edema and the marked reduction of the gray matter/white matter ratio (GWR) on CT obtained within 2 hours after ROSC can predict poor neurologic outcomes with 0\% FPR in comatose patients who received TTM after cardiac arrest. However, different studies have reported different GWR thresholds with $0 \%$ FPR, depending on the measurement technique and the studied brain area. ${ }^{77,98,99}$ An extensive restriction of diffusion detected on brain magnetic resonance imaging (MRI) obtained 2 to 6 days after ROSC and quantitatively measured with an apparent diffusion coefficient can predict poor neurologic outcomes with 0\% FPR in comatose patients who received TM after cardiac arrest. ${ }^{100,101}$ However, the apparent diffusion coefficient threshold value with 0\% FPR is different among studies, depending on the studied brain area and the measurement technique.

Therefore, it is recommended to use the marked reduction of the GWR on CT obtained within 2 hours after ROSC or the extensive restriction of diffusion on MRI obtained 2 to 6 days after ROSC in combination with other predictors to predict poor neurologic outcomes of comatose patients who received TTM after cardiac arrest.

\section{Neurological prognostication algorithm (Fig. 2)}

In most post-cardiac arrest comatose patients, cerebral function recovery occurs within 72 hours after ROSC. ${ }^{84,102}$ However, there is a possibility of decreased accuracy of clinical examination due to the influence of sedatives and neuromuscular blockers administered during the application of TM.

First, it is important to exclude confounders (e.g., residual sedation and paralysis) that can impact clinical examination after 72 hours after ROSC and the completion of TM. Second, 72 hours after ROSC, use the flowchart to differentiate comatose patients who show the absence of motor movement or extensor posturing to pain. Third, confirm the bilateral absence of pupillary light reflex and bilateral absence of N2O waveform in SSEPs, which have a low FPR and high accuracy. If all three aforementioned conditions are met, a poor neurological outcome can be predicted with accuracy (FPR $<5 \%$, narrow 95\% confidence interval).

Otherwise, observe patients for at least 24 hours and assess the following predictors of poor outcome. 1) A marked reduction of the GWR on brain CT obtained within 2 hours after ROSC; 2) the lack of normal trace in the amplitude-integrated EEG recording observed for $>36$ hours; 3 ) status myoclonus occurring within 72 hours after ROSC; 4) a high serum level of NSE measured 48 to 72 hours after the ROSC; 5) unreactive burst-suppression or status epilepticus on EEG 72 hours after ROSC; and 6) extensive restriction of diffusion on MRI obtained 2 to 6 days after
ROSC. A poor neurologic outcome can be predicted if two of six factors are observed. Otherwise, the outcome can be determined based on various findings in combination or after additional observation.

\section{ACKNOWLEDGMENTS}

We appreciate to the following evidence reviewers who contribute to develop the 2015 Korean Consensus on Cardiopulmonary Resuscitation and Emergency Cardiovascular Care Science with Treatment Recommendations for the population, intervention, comparator, and outcome questions in post-cardiac arrest care. The collaborators' are as follows: Kyung Su Kim, Seoul National University Hospital, Seoul, Korea; Won Young Kim, Ulsan University Asan Medical Center, Seoul, Korea; Jin Joo Kim, Gachon University Gil Medical Center, Incheon, Korea; Min Seob Sim, Sungkyunkwan University Samsung Medical Center, Seoul, Korea; Yeon Ho You, Chungnam National University Hospital, Kwangju, Korea; Seung Joon Lee, Myongji Hospital, Seoul, Korea; Young Hwan Lee, Hallym University Sacred Heart Hospital, Seoul, Korea; Joo Young Lee, The Catholic University of Korea Seoul St. Mary's Hospital, Seoul, Korea; Kyung Woon Jeung, Chonnam National University Hospital, Kwangju, Korea; Moon Gu Han, Seoul National University Bundang Hospital, Seongnam, Korea; Chul Han, Ewha University Hospital, Seoul, Korea.

\section{REFERENCES}

1. Kilgannon JH, Jones $A E$, Shapiro $\mathrm{NI}$, et al. Association between arterial hyperoxia following resuscitation from cardiac arrest and in-hospital mortality. JAMA 2010;303:216571.

2. Kilgannon JH, Jones AE, Parrillo JE, et al. Relationship between supranormal oxygen tension and outcome after resuscitation from cardiac arrest. Circulation 2011;123:271722.

3. Janz DR, Hollenbeck RD, Pollock JS, McPherson JA, Rice TW. Hyperoxia is associated with increased mortality in patients treated with mild therapeutic hypothermia after sudden cardiac arrest. Crit Care Med 2012;40:3135-9.

4. Bellomo R, Bailey M, Eastwood GM, et al. Arterial hyperoxia and in-hospital mortality after resuscitation from cardiac arrest. Crit Care 2011;15:R90.

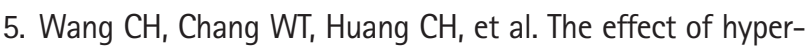
oxia on survival following adult cardiac arrest: a systematic review and meta-analysis of observational studies. Resuscitation 2014;85:1142-8. 
6. Menon DK, Coles JP, Gupta AK, et al. Diffusion limited oxygen delivery following head injury. Crit Care Med 2004;32: 1384-90.

7. Schneider AG, Eastwood GM, Bellomo R, et al. Arterial carbon dioxide tension and outcome in patients admitted to the intensive care unit after cardiac arrest. Resuscitation 2013;84:927-34.

8. Roberts BW, Kilgannon JH, Chansky ME, Mittal N, Wooden J, Trzeciak S. Association between postresuscitation partial pressure of arterial carbon dioxide and neurological outcome in patients with post-cardiac arrest syndrome. Circulation 2013;127:2107-13.

9. Vaahersalo J, Bendel S, Reinikainen M, et al. Arterial blood gas tensions after resuscitation from out-of-hospital cardiac arrest: associations with long-term neurologic outcome. Crit Care Med 2014;42:1463-70.

10. Lee BK, Jeung KW, Lee HY, et al. Association between mean arterial blood gas tension and outcome in cardiac arrest patients treated with therapeutic hypothermia. Am J Emerg Med 2014;32:55-60.

11. Laurent I, Monchi M, Chiche JD, et al. Reversible myocardial dysfunction in survivors of out-of-hospital cardiac arrest. J Am Coll Cardiol 2002;40:2110-6.

12. Kern KB, Hilwig RW, Berg RA, et al. Postresuscitation left ventricular systolic and diastolic dysfunction. Treatment with dobutamine. Circulation 1997;95:2610-3.

13. Manzo-Silberman S, Fichet J, Mathonnet $A$, et al. Percutaneous left ventricular assistance in post cardiac arrest shock: comparison of intra aortic blood pump and IMPELLA Recover LP2.5. Resuscitation 2013;84:609-15.

14. Trzeciak $\mathrm{S}$, Jones $A E_{\text {, Kilgannon } J H}$, et al. Significance of arterial hypotension after resuscitation from cardiac arrest. Crit Care Med 2009;37:2895-903.

15. Bray JE, Bernard S, Cantwell K, Stephenson M, Smith K; VACAR Steering Committee. The association between systolic blood pressure on arrival at hospital and outcome in adults surviving from out-of-hospital cardiac arrests of presumed cardiac aetiology. Resuscitation 2014;85:509-15.

16. Kilgannon JH, Roberts BW, Reihl LR, et al. Early arterial hypotension is common in the post-cardiac arrest syndrome and associated with increased in-hospital mortality. Resuscitation 2008;79:410-6.

17. Gaieski DF, Band RA, Abella BS, et al. Early goal-directed hemodynamic optimization combined with therapeutic hypothermia in comatose survivors of out-of-hospital cardiac arrest. Resuscitation 2009;80:418-24.

18. Sunde $K$, Pytte $M$, Jacobsen $D$, et al. Implementation of a standardised treatment protocol for post resuscitation care after out-of-hospital cardiac arrest. Resuscitation 2007;73: 29-39.

19. Orioles A, Morrison WE, Rossano JW, et al. An under-recognized benefit of cardiopulmonary resuscitation: organ transplantation. Crit Care Med 2013;41:2794-9.

20. Walters EL, Morawski K, Dorotta I, et al. Implementation of a post-cardiac arrest care bundle including therapeutic hypothermia and hemodynamic optimization in comatose patients with return of spontaneous circulation after out-ofhospital cardiac arrest: a feasibility study. Shock 2011;35: 360-6.

21. Mullner $M$, Sterz $F$, Binder $M$, et al. Arterial blood pressure after human cardiac arrest and neurological recovery. Stroke 1996;27:59-62.

22. Kilgannon JH, Roberts BW, Jones $A E$, et al. Arterial blood pressure and neurologic outcome after resuscitation from cardiac arrest. Crit Care Med 2014;42:2083-91.

23. Myerburg RJ, Kessler KM, Castellanos A. Sudden cardiac death: epidemiology, transient risk, and intervention assessment. Ann Intern Med 1993;119:1187-97.

24. Adabag AS, Therneau TM, Gersh BJ, Weston SA, Roger VL. Sudden death after myocardial infarction. JAMA 2008;300: 2022-9.

25. Authors/Task Force Members, Priori SG, Blomstrom-Lundqvist C, et al. 2015 ESC Guidelines for the management of patients with ventricular arrhythmias and the prevention of sudden cardiac death: The Task Force for the Management of Patients with Ventricular Arrhythmias and the Prevention of Sudden Cardiac Death of the European Society of Cardiology (ESC)Endorsed by: Association for European Paediatric and Congenital Cardiology (AEPC). Europace 2015;17:160187.

26. Jacobs AK. Regional systems of care for patients with ST-elevation myocardial infarction: being at the right place at the right time. Circulation 2007;116:689-92.

27. Strote JA, Maynard C, Olsufka $M$, et al. Comparison of role of early (less than six hours) to later (more than six hours) or no cardiac catheterization after resuscitation from outof-hospital cardiac arrest. Am J Cardiol 2012;109:451-4.

28. Nanjayya VB, Nayyar V. Immediate coronary angiogram in comatose survivors of out-of-hospital cardiac arrest: an Australian study. Resuscitation 2012;83:699-704.

29. Zanuttini D, Armellini I, Nucifora G, et al. Impact of emergency coronary angiography on in-hospital outcome of unconscious survivors after out-of-hospital cardiac arrest. Am J Cardiol 2012;110:1723-8. 
30. Hollenbeck RD, McPherson JA, Mooney MR, et al. Early cardiac catheterization is associated with improved survival in comatose survivors of cardiac arrest without STEMI. Resuscitation 2014;85:88-95.

31. Bro-Jeppesen J, Kjaergaard J, Wanscher M, et al. Emergency coronary angiography in comatose cardiac arrest patients: do real-life experiences support the guidelines? Eur Heart J Acute Cardiovasc Care 2012;1:291-301.

32. Scholz KH, Hilmer T, Schuster S, Wojcik J, Kreuzer H, Tebbe U. Thrombolysis in resuscitated patients with pulmonary embolism. Dtsch Med Wochenschr 1990;115:930-5.

33. Ullmann $M$, Hemmer W, Hannekum A. The urgent pulmonary embolectomy: mechanical resuscitation in the operating theatre determines the outcome. Thorac Cardiovasc Surg 1999;47:5-8.

34. Konstantinov IE, Saxena P, Koniuszko MD, Alvarez J, Newman MA. Acute massive pulmonary embolism with cardiopulmonary resuscitation: management and results. Tex Heart Inst J 2007;34:41-5.

35. Zeiner A, Holzer M, Sterz F, et al. Hyperthermia after cardiac arrest is associated with an unfavorable neurologic outcome. Arch Intern Med 2001;161:2007-12.

36. Gebhardt K, Guyette FX, Doshi AA, Callaway CW, Rittenberger JC; Post Cardiac Arrest Service. Prevalence and effect of fever on outcome following resuscitation from cardiac arrest. Resuscitation 2013;84:1062-7.

37. Leary $M$, Grossestreuer $A V$, lannacone $S$, et al. Pyrexia and neurologic outcomes after therapeutic hypothermia for cardiac arrest. Resuscitation 2013;84:1056-61.

38. Bro-Jeppesen J, Hassager C, Wanscher M, et al. Post-hypothermia fever is associated with increased mortality after out-of-hospital cardiac arrest. Resuscitation 2013;84:173440.

39. Badjatia N. Hyperthermia and fever control in brain injury. Crit Care Med 2009;37(7 Suppl):S250-7.

40. Bohman LE, Levine JM. Fever and therapeutic normothermia in severe brain injury: an update. Curr Opin Crit Care 2014; 20:182-8.

41. Hypothermia after Cardiac Arrest Study Group. Mild therapeutic hypothermia to improve the neurologic outcome after cardiac arrest. N Engl J Med 2002;346:549-56.

42. Bernard SA, Gray TW, Buist MD, et al. Treatment of comatose survivors of out-of-hospital cardiac arrest with induced hypothermia. N Engl J Med 2002;346:557-63.

43. Testori $C$, Sterz F, Behringer $W$, et al. Mild therapeutic hypothermia is associated with favourable outcome in patients after cardiac arrest with non-shockable rhythms. Resuscita- tion 2011;82:1162-7.

44. Dumas F, Grimaldi $D$, Zuber B, et al. Is hypothermia after cardiac arrest effective in both shockable and nonshockable patients?: insights from a large registry. Circulation 2011;123: 877-86.

45. Vaahersalo J, Hiltunen $\mathrm{P}$, Tiainen $\mathrm{M}$, et al. Therapeutic hypothermia after out-of-hospital cardiac arrest in Finnish intensive care units: the FINNRESUSCI study. Intensive Care Med 2013;39:826-37.

46. Donnino MW, Andersen LW, Berg KM, et al. Temperature Management After Cardiac Arrest: An Advisory Statement by the Advanced Life Support Task Force of the International Liaison Committee on Resuscitation and the American Heart Association Emergency Cardiovascular Care Committee and the Council on Cardiopulmonary, Critical Care, Perioperative and Resuscitation. Circulation 2015;132:2448-56.

47. Nichol $G$, Huszti $E_{1}$ Kim F, et al. Does induction of hypothermia improve outcomes after in-hospital cardiac arrest? Resuscitation 2013;84:620-5.

48. Nielsen N, Wetterslev J, Cronberg T, et al. Targeted temperature management at $33^{\circ} \mathrm{C}$ versus $36^{\circ} \mathrm{C}$ after cardiac arrest. N Engl J Med 2013;369:2197-206.

49. Ginsberg MD, Belayev L. Biological and molecular mechanisms of hypothermic neuroprotection. In: Mayer SA, Sessler DI. Therapeutic hypothermia. New York, NY: Marcel Dekker; 2005. p.85-140.

50. Liu L, Yenari MA. Therapeutic hypothermia: neuroprotective mechanisms. Front Biosci 2007;12:816-25.

51. Angelos MG, Menegazzi JJ, Callaway CW. Bench to bedside: resuscitation from prolonged ventricular fibrillation. Acad Emerg Med 2001;8:909-24.

52. van Zanten $A R$, Polderman KH. Early induction of hypothermia: will sooner be better? Crit Care Med 2005;33:1449-52.

53. Kim F, Olsufka $\mathrm{M}$, Longstreth WT Jr, et al. Pilot randomized clinical trial of prehospital induction of mild hypothermia in out-of-hospital cardiac arrest patients with a rapid infusion of 4 degrees $C$ normal saline. Circulation 2007;115:3064-70.

54. Kamarainen A, Virkkunen I, Tenhunen J, Yli-Hankala A, Silfvast T. Prehospital therapeutic hypothermia for comatose survivors of cardiac arrest: a randomized controlled trial. Acta Anaesthesiol Scand 2009;53:900-7.

55. Bernard SA, Smith K, Cameron P, et al. Induction of therapeutic hypothermia by paramedics after resuscitation from out-of-hospital ventricular fibrillation cardiac arrest: a randomized controlled trial. Circulation 2010;122:737-42.

56. Castren $M$, Nordberg $P$, Svensson L, et al. Intra-arrest transnasal evaporative cooling: a randomized, prehospital, multi- 
center study (PRINCE: Pre-ROSC IntraNasal Cooling Effectiveness). Circulation 2010;122:729-36.

57. Bernard SA, Smith K, Cameron P, et al. Induction of prehospital therapeutic hypothermia after resuscitation from nonventricular fibrillation cardiac arrest. Crit Care Med 2012; 40:747-53.

58. Kim F, Nichol G, Maynard C, et al. Effect of prehospital induction of mild hypothermia on survival and neurological status among adults with cardiac arrest: a randomized clinical trial. JAMA 2014;311:45-52.

59. Debaty G, Maignan M, Savary D, et al. Impact of intra-arrest therapeutic hypothermia in outcomes of prehospital cardiac arrest: a randomized controlled trial. Intensive Care Med 2014;40:1832-42.

60. Geocadin RG, Koenig MA, Jia X, Stevens RD, Peberdy MA. Management of brain injury after resuscitation from cardiac arrest. Neurol Clin 2008;26:487-506.

61. Shin J, Kim J, Song K, Kwak Y. Core temperature measurement in therapeutic hypothermia according to different phases: comparison of bladder, rectal, and tympanic versus pulmonary artery methods. Resuscitation 2013;84:810-7.

62. Oksanen T, Skrifvars MB, Varpula T, et al. Strict versus moderate glucose control after resuscitation from ventricular fibrillation. Intensive Care Med 2007;33:2093-100.

63. Marik PE, Preiser JC. Toward understanding tight glycemic control in the ICU: a systematic review and metaanalysis. Chest 2010;137:544-51.

64. Wiener RS, Wiener DC, Larson RJ. Benefits and risks of tight glucose control in critically ill adults: a meta-analysis. JAMA 2008;300:933-44.

65. Rittenberger JC, Popescu A, Brenner RP, Guyette FX, Callaway $\mathrm{CW}$. Frequency and timing of nonconvulsive status epilepticus in comatose post-cardiac arrest subjects treated with hypothermia. Neurocrit Care 2012;16:114-22.

66. Caviness JN, Brown P. Myoclonus: current concepts and recent advances. Lancet Neurol 2004;3:598-607.

67. Krumholz A, Stern BJ, Weiss HD. Outcome from coma after cardiopulmonary resuscitation: relation to seizures and myoclonus. Neurology 1988;38:401-5.

68. Hui AC, Cheng C, Lam A, Mok V, Joynt GM. Prognosis following Postanoxic Myoclonus Status epilepticus. Eur Neurol 2005;54:10-3.

69. Chamorro C, Borrallo JM, Romera MA, Silva JA, Balandin B. Anesthesia and analgesia protocol during therapeutic hypothermia after cardiac arrest: a systematic review. Anesth Analg 2010;110:1328-35.

70. Salciccioli JD, Cocchi MN, Rittenberger JC, et al. Continuous neuromuscular blockade is associated with decreased mortality in post-cardiac arrest patients. Resuscitation 2013;84: 1728-33.

71. Samaniego EA, Mlynash M, Caulfield AF, Eyngorn I, Wijman CA. Sedation confounds outcome prediction in cardiac arrest survivors treated with hypothermia. Neurocrit Care 2011; 15:113-9.

72. Zandbergen EG, Hijdra A, Koelman JH, et al. Prediction of poor outcome within the first 3 days of postanoxic coma. Neurology 2006;66:62-8.

73. Bisschops $L L$, van Alfen $N$, Bons $S$, van der Hoeven JG, Hoedemaekers $\mathrm{CW}$. Predictors of poor neurologic outcome in patients after cardiac arrest treated with hypothermia: a retrospective study. Resuscitation 2011;82:696-701.

74. Bouwes A, Binnekade JM, Zandstra DF, et al. Somatosensory evoked potentials during mild hypothermia after cardiopulmonary resuscitation. Neurology 2009;73:1457-61.

75. Bouwes A, Binnekade JM, Kuiper MA, et al. Prognosis of coma after therapeutic hypothermia: a prospective cohort study. Ann Neurol 2012;71:206-12.

76. Fugate JE, Wijdicks EF, Mandrekar J, et al. Predictors of neurologic outcome in hypothermia after cardiac arrest. Ann Neurol 2010;68:907-14.

77. Wu O, Batista LM, Lima FO, Vangel MG, Furie KL, Greer DM. Predicting clinical outcome in comatose cardiac arrest patients using early noncontrast computed tomography. Stroke 2011;42:985-92.

78. Greer DM, Yang J, Scripko PD, et al. Clinical examination for prognostication in comatose cardiac arrest patients. Resuscitation 2013;84:1546-51.

79. Dragancea I, Horn J, Kuiper M, et al. Neurological prognostication after cardiac arrest and targeted temperature management $33^{\circ} \mathrm{C}$ versus $36^{\circ} \mathrm{C}$ : results from a randomised controlled clinical trial. Resuscitation 2015;93:164-70.

80. Rossetti AO, Oddo M, Logroscino G, Kaplan PW. Prognostication after cardiac arrest and hypothermia: a prospective study. Ann Neurol 2010;67:301-7.

81. Rossetti AO, Urbano LA, Delodder F, Kaplan PW, Oddo M. Prognostic value of continuous EEG monitoring during therapeutic hypothermia after cardiac arrest. Crit Care 2010;14: R173.

82. Rossetti AO, Carrera E, Oddo M. Early EEG correlates of neuronal injury after brain anoxia. Neurology 2012;78:796-802.

83. Bouwes $A$, van Poppelen $D$, Koelman JH, et al. Acute posthypoxic myoclonus after cardiopulmonary resuscitation. BMC Neurol 2012;12:63.

84. Wijdicks EF, Young GB. Myoclonus status in comatose pa- 
tients after cardiac arrest. Lancet 1994;343:1642-3.

85. Stelzl T, von Bose MJ, Hogl B, Fuchs HH, Flugel KA. A comparison of the prognostic value of neuron-specific enolase serum levels and somatosensory evoked potentials in 13 reanimated patients. Eur J Emerg Med 1995;2:24-7.

86. Tiainen M, Kovala $\Pi$, Takkunen OS, Roine RO. Somatosensory and brainstem auditory evoked potentials in cardiac arrest patients treated with hypothermia. Crit Care Med 2005; 33:1736-40.

87. Zingler VC, Krumm B, Bertsch T, Fassbender K, PohlmannEden B. Early prediction of neurological outcome after cardiopulmonary resuscitation: a multimodal approach combining neurobiochemical and electrophysiological investigations may provide high prognostic certainty in patients after cardiac arrest. Eur Neurol 2003;49:79-84.

88. Rothstein TL. The role of evoked potentials in anoxic-ischemic coma and severe brain trauma. J Clin Neurophysiol 2000;17:486-97.

89. Zanatta P, Messerotti Benvenuti S, Baldanzi F, Bosco E. Painrelated middle-latency somatosensory evoked potentials in the prognosis of post anoxic coma: a preliminary report. Minerva Anestesiol 2012;78:749-56.

90. Crepeau AZ, Rabinstein AA, Fugate JE, et al. Continuous EEG in therapeutic hypothermia after cardiac arrest: prognostic and clinical value. Neurology 2013;80:339-44.

91. Oddo M, Rossetti AO. Early multimodal outcome prediction after cardiac arrest in patients treated with hypothermia. Crit Care Med 2014;42:1340-7.

92. Wennervirta JE, Ermes MJ, Tiainen SM, et al. Hypothermiatreated cardiac arrest patients with good neurological outcome differ early in quantitative variables of EEG suppression and epileptiform activity. Crit Care Med 2009;37:242735.

93. Kawai M, Thapalia U, Verma A. Outcome from therapeutic hypothermia and EEG. J Clin Neurophysiol 2011;28:483-8.

94. Oh SH, Park KN, Kim YM, et al. The prognostic value of con- tinuous amplitude-integrated electroencephalogram applied immediately after return of spontaneous circulation in therapeutic hypothermia-treated cardiac arrest patients. Resuscitation 2013;84:200-5.

95. Oh SH, Park KN, Shon YM, et al. Continuous amplitude-integrated electroencephalographic monitoring is a useful prognostic tool for hypothermia-treated cardiac arrest patients. Circulation 2015;132:1094-103.

96. Oksanen T, Tiainen M, Skrifvars MB, et al. Predictive power of serum NSE and OHCA score regarding 6-month neurologic outcome after out-of-hospital ventricular fibrillation and therapeutic hypothermia. Resuscitation 2009;80:16570.

97. Rundgren $M$, Karlsson $T$, Nielsen $N$, Cronberg $T$, Johnsson $P_{\text {, }}$ Friberg $\mathrm{H}$. Neuron specific enolase and $\mathrm{S}-100 \mathrm{~B}$ as predictors of outcome after cardiac arrest and induced hypothermia. Resuscitation 2009;80:784-9.

98. Lee BK, Jeung KW, Lee HY, Jung YH, Lee DH. Combining brain computed tomography and serum neuron specific enolase improves the prognostic performance compared to either alone in comatose cardiac arrest survivors treated with therapeutic hypothermia. Resuscitation 2013;84:1387-92.

99. Kim SH, Choi SP, Park KN, Youn CS, Oh SH, Choi SM. Early brain computed tomography findings are associated with outcome in patients treated with therapeutic hypothermia after out-of-hospital cardiac arrest. Scand J Trauma Resusc Emerg Med 2013;21:57.

100. Els T, Kassubek J, Kubalek R, Klisch J. Diffusion-weighted MRI during early global cerebral hypoxia: a predictor for clinical outcome? Acta Neurol Scand 2004;110:361-7.

101. Mlynash M, Campbell DM, Leproust EM, et al. Temporal and spatial profile of brain diffusion-weighted MRI after cardiac arrest. Stroke 2010;41:1665-72.

102. Jorgensen EO, Holm S. The natural course of neurological recovery following cardiopulmonary resuscitation. Resuscitation 1998;36:111-22. 